Revue internationale P.M.E.

Économie et gestion de la petite et moyenne entreprise

\title{
Le développement local : un intérêt qui n'en démord pas
}

\section{André Joyal}

Volume 3, numéro 2, 1990

URI : https://id.erudit.org/iderudit/1007980ar

DOI : https://doi.org/10.7202/1007980ar

Aller au sommaire du numéro

Éditeur(s)

Presses de l’Université du Québec

ISSN

0776-5436 (imprimé)

1918-9699 (numérique)

Découvrir la revue

Citer ce document

Joyal, A. (1990). Le développement local : un intérêt qui n'en démord pas. Revue internationale P.M.E., 3(2), 249-256. https://doi.org/10.7202/1007980ar d'utilisation que vous pouvez consulter en ligne.

https://apropos.erudit.org/fr/usagers/politique-dutilisation/ 


\title{
Notes de lecture Le développement local : un intérêt qui n'en démord pas
}

\author{
André JOYAL* \\ GREPME \\ Université du Québec à Trois-Rivières
}

La crise de la régulation fordiste a incité, d'abord en milieu rural, ensuite en milieu urbain, les collectivités locales à assumer une plus grande part de responsabilité dans le développement de leur environnement immédiat. Depuis le milieu des années 1980, l'abondance d'ouvrages et d'articles sur le développement local est à la mesure des espoirs véhiculés par les partisans d'une stratégie économique centrée autour de la mise en valeur des ressources humaines, financières et physiques d'un milieu donné. Cette note a pour objectif d'éclaircir la question tout en présentant une variété de contributions émanant des deux côtés de l'Atlantique et susceptibles de fournir aux lecteurs intéressés une source précieuse d'informations et de réflexions.

\section{Définition}

Le développement local, faut-il s'en surprendre, soulève chez leséconomistes des positions différentes, voire opposées et surtout bon nombre d'interrogations. Dans un numéro intitulé Les territoires incertains du local, Les Cahiers du LERASS (1990) s'interrogent sur l'opportunité de repenser le local. Alors que dans un ouvrage

* André Joyal est docteur en sciences économiques de l'Université de Dijon. Il est professeur à l'Université du Québec à Trois-Rivières. Il a produit plusieurs ouvrages sur l'économie altemative. Adresse: GREPME, Université du Québec à Trois-Rivières, C.P. 500, Trois-Rivières, G9A 5H7. 
des plus stimulants sur le sujet, et que l'on ne peut trop recommander, E. J. Blakeley (1989) considère que les termes «régional» et «local» sont interchangeables dans la mesure où l'on se rapporte à une ère géographique, caractérisée par des intérêts communs et placée sous l'autorité d'un ou de plusieurs gouvernements locaux. A l'instar des bassins d'emplois français, les habitants d'une telle zone répondent à leurs besoins de travail, d'achats et de loisirs sans déplacements importants. Comme d'autres auteurs, ce professeur d'économie rurale et urbaine à l'Université de la Californie à Berkeley estime que le développement économique local correspond davantage à un mouvement qu'à un modèle économique bien structuré duquel émerge une approche clairement définie.

En fait, c'est l'importance accordée aux politiques macro-économiques et aux méga-projets par une majorité d'économistes qui suscite le scepticisme et incite certains à favoriser l'approche du développement local. Ces derniers, parmi les disciples d'Adam Smith, feraient partie d'une espèce fort peu répandue, si l'on en croit Marie-Élisabeth Chassagne, l'un des principaux thuriféraires de «l'école française» du développement «par le bas». «Pour les économistes : le développement local n'existe pas», écrit-elle dans les forts intéressants actes de la deuxième rencontre d'Aurillac (1987) préfacés par Jacques Delors. Selon l'auteur, les économistes (appartenant au courant dominant) réfutent la notion même de développement localisé en privilégiant l'étude des déterminants de la localisation. Heureusement, la confrérie ne représente pas un bloc monolithique; aussi, certains de ses membres se retrouvent en compagnie de sociologues, de géographes, de spécialistes de la gestion, d'urbanistes, etc., afin de traiter d'un développement faisant appel à des initiatives issues d'une démarche partenariale. Sur ces dernières, le lecteur profitera des pénétrantes réflexions de Maurice Allefresde qui, de façon très pertinente, évite le piège de l'association du développement local à la recherche d'autarcie. Au contraire, pour une collectivité territoriale donnée, il souhaite le recours aux ressources complémentaires des collectivités voisines.

Par ailleurs, force est de le reconnaître, malgré (ou à cause de) l'abondance des écrits, colloques, conférences ou autres symposiums sur la problématique du développement local, que le concept demeure ambigu. Dans un ouvrage collectif comprenant pas moins de vingt-sept contributions, Hugues Dionne (1989) cite J. Arocena (1986), pour qui il n'y a pas une théorie sur le développement local, mais des théories sur le développement qui different entre elles dans la façon de considérer le «local». H. Dionne signale le danger d'un discours ayant tendance à homogénéiser les situations spécifiques et différenciées de développement des territoires locaux, qu'il s'agisse de quartiers urbains, d'agglomérations de banlieues, de paroisses périurbaines ou de villages ruraux périphériques. De façon fort pertinente, ce chercheur de l'Université du Québec à Rimouski poursuit ses mises en garde contre l'engouement actuel en faveur de l'entrepreneuriat local de la part de l'Etat. En effet, les politiques économiques qui en découlent ont tendanceà privilégier une approche technocratique du développement local et de l'espace fortement marquée par une logique strictement économique aux détriments de celle d'un développement local communautaire 
centré sur un espace habité, construit sur une identité sociale et culturelle à enrichir. Gregory MacLeod (1989), dans un autre ouvrage collectif faisant appel à des auteurs de six pays différents, partage ce point de vue sur la base d'expériences vécues dans l'Est du Canada. Il distingue deux types d'organismes de soutien à la création d'entreprise. Certains émanent de différents programmes d'interventions gouvernementales, alors que d'autres proviennent avant tout d'une volonté des différents acteurs du milieu. Ces derniers obtiennent évidemment la préférence de l'auteur.

Il importe donc de retenir que si le développement local suscite la création de petites entreprises, toute création de celles-ci ne résulte pas nécessairement d'une démarche associée au développement local. En conséquence, l'émergence de PME et le développement local ne sont pas nécessairement synonymes. Des petites régions peuvent se distinguer par un dynamisme fortement identifié par l'essor de PME sans qu'elles aient fait appel à une stratégie de développement local.

La référence aux efforts d'émancipation économique decertainescollectivités du Bas-Saint-Laurent et de la Gaspésie, au Québec, à partir du milieu des années 1970, aide à saisir la nuance. Inspirée par d'heureux résultats, obtenus aux Etats-Unis dans un environnement défavorisé, suite à la mise en oeuvre d'une stratégie appelée community economic development, les populations de certaines municipalités ont réagi face à la précarité de leur situation. On se rapporte ici à des initiatives dont la finalité marchande s'accompagne d'une finalité sociale bien marquée de manière à faire de l'entreprise un moyen d'émancipation économique et un outil de mieux-être pour ses travailleurs. C'est pourquoi les expressions «développement local» et «développement communautaire» se confondent souvent au Québec.

\section{L'interrelation entre le social et l'économique}

A Bruxelles, la Direction générale de l'emploi, des affaires sociales et de l'éducation de la Commission des Communautés Européennes montre un intérêt certain envers le sujet qui nous préoccupe. Dans les notes de synthèse d'un cycle de douze séminaires (1989), on lit : «C'est dans le contexte de remise en cause des systèmes classiques que s'inscrit aujourd'hui le débat sur la place et le rôle qu'il faut accorder à la problématique locale.» Il est fait allusion à une nouvelle approche prenant appui sur trois exigences fondamentalement innovantes eu égard à la réalité sociale : la prise en compte des groupes et des personnes qui la composent; la mise en oeuvre d'une démarche adaptée aux problèmes identifiés au niveau local; l'association étroite des volets sociaux et économiques dans une perspective de développement local. Il est précisé que dans cette nouvelle optique, «local» ne signifie plus «territoire administrativement limité». La dimension locale devient celle d'un espace propre à la prise de conscience et aux potentialités d'entreprendre. Nous retrouvons l'allusion à la conjugaison des intérêts convergents. D'intéressantes considérations se rapportent au rôle-clé dévolu aux agents de développement investis de la responsabilité d'accueillir, de conseiller, d'aider les nouveaux entrepreneurs en 
plus de voir à la coordination des efforts des différents acteurs locaux. Aux institutions de formation en gestion et en économique d'assurer la formation de ce genre d'intervenant.

Un rapport d'étape, issu des travaux de chercheurs français membres du Groupe Interministériel sur le Développement Economique Local (GIDEL, 1989), souligne à son tour l'importance du soutien à l'entreprise. En effet, l'importance de l'encadrement des créateurs d'entreprise est mise en évidence par une allusion au taux d'échecs qui passerait de $50 \%$ à $20 \%$, suite à la participation des futurs entrepreneurs à une session d'initiation à la conduite d'un projet d'entreprise. Les autorités gouvernementales doivent fournir, sur une base temporaire, et de concert avec d'autres acteurs, les appuis indispensables aux initiatives locales. Pour ce groupe de réflexion, le développement économique local se situe à trois niveaux :

1. celui de l'entreprise : on se rapporte ici à des emplois adaptés à la main-d'oeuvre locale et offrant des possibilités d'insertion pour les chômeurs de longue durée;

2. celui de l'action collective globale : on pense cette fois à la mobilisation des différents partenaires, publics et privées, autour d'un projet d'ensemble appuyé sur des activités économiques;

3. les échelons régional et national : sont concernées ici les différentes politiques d'appui en matière de financement des entreprises, de conseil, de transferts de technologie et de formation.

L'opportunité de favoriser le développement de réseaux d'appui aux petites entreprises mérite deux mentions. L'importance d'une certaine dynamique locale susceptible de favoriser l'émergence d'activités économiques durables se trouve ainsi reconnue.

De son côté, l'OCDE ne fait pas bande à part. En effet, depuis 1982, dans le cadre de son programme «Initiatives locales de création d'emplois (ILE)», un grand nombre de documents publiés régulièrement par l'organisme du château de la Muette offrent une manne précieuse d'information surle développement social etéconomique de chacun des pays membres. Publié conjointement avec la Commission des Communautés européennes, le périodique Innovation et Emploi traite d'un sujet particulier dans chaque numéro en plus d'informer sur les parutions récentes et les différentes conférences et rencontres internationales ayant trait à la dynamique locale. Diffuséesà une plus petite échelle, les Notes du Secrétariat du programme ILE fournissent de l'information sur une gamme variée de sujets comme les jeunes entrepreneurs, les femmes entrepreneures, les chômeurs créateurs d'entreprise, les organismes de soutien, les initiatives économiques en milieu rural. Ces dernières ne peuvent manquer d'intéresser ceux qui se préoccupent du maintien en vie de régions dites éloignées ou périphériques. Si le développement local ou communautaire a connu ses premières expériences dans différents «arrières-pays» durant les années 1970, sa démarche demeure toujours pertinente face à la menace de désertification de zones fragilisées par la crise économique. 


\section{Le développement local en milieu rural}

En France, par exemple, la référence au rural se comprend aisément par le fait que $90 \%$ des 37000 communes ont moins de 2000 habitants et sont ainsi considérées comme rurales. Quel développement économique faut-il favoriser si l'on précise que $31 \%$ de ces communes ont moins de 200 habitants ? Un projet s'impose.

Une brochette de représentants d'une certaine gauche a relevé le défí en publiant «Le projet rural» (1988). Nous sommes loin du projet socialiste de 1980 qui dénonçait l'idéologie dominante pour son éloge du risque, de l'initiative et de la responsabilité du chef d'entreprise. Dix ans ont passé. On dirait un siècle à en juger par les réflexions de ceux qui ne font plus leurs choux gras des vieilles maximes idéologiques. Il faut reconnaître que, déjà en 1984, les intentions de rupture avec le capitalisme avaient laissé place à la reconnaissance des vertus del'initiative individuelle et des avantages du marché.

Dans la préface, à propos du défi de l'emploi, Pierre Mauroy fait allusion aux entreprises intermédiaires (appelées maintenant entreprises d'insertion de travailleurs en marge du marché régulier du travail), en vue de répondre à un intérêt général négligé autant par les collectivités locales que par le secteur privé. La recherche du meilleur échelon d'intervention possible est également présenté comme l'un des principaux thèmes de réflexion. Tous auront deviné qu'il s'agit de l'échelon local. Pour sa part, l'ancien ministre et maire d'Aurillac, René Souchon, précise bien l'enjeu du débat en considérant que le projet pour la France rurale, c'est d'abord un état d'esprit, une volonté de jouer toutes les initiatives dans le cadre du partenariat et de la solidarité organisée. De son côté, fier d'une première expérience à la tête du ministère de l'Emploi et des Affaires sociales, Michel Delebarre, face à la nécessité de moderniser le secteur de l'agro-alimentaire, suggère une politique concertée de l'emploi, en milieu rural, à trois volets. L'un d'entre eux nous est maintenant familier, puisqu'il se rapporte à l'accompagnement de l'emploi par l'innovation : formation, conseil, transfert de technologie, innovation pour l'insertion des jeunes, partenariat pour rompre l'isolement des dirigeants d'entreprise et faciliter les conceptions de politiques locales pour l'emploi. D'autres contributions sont l'oeuvre d'experts «tenus à l'obligation de réserve». L'une d'elle ne suggère rien de moins que l'association des entrepreneurs et des banquiers dans un processus partenarial. Il y a un commencement à tout. Mais fallait-il vraiment le voile de l'anonymat pour avancer une telle évidence ? L'idée est lancée. Laissons le temps faire son oeuvre. Enfin, les représentants de la société civile, dans ce bel aréopage, ne sont heureusement pas absents. Ainsi, l'on retrouve Marie-Élisabeth Chassagne, à l'époque présidente de la Commission ruralité du Groupe de recherche pour l'Education et la Prospective, qui attire l'attention sur les opportunités des nouvelles technologies en régions périphériques ou éloignées des grands centres. «Au fond de la Creuse un atelier tout neuf emploie 20 personnes à la fabrication de codeurs optiques pour l'industrie de l'armement. C'est la haute technologie aux champs.» 
L'idée que la technologie puisse servir à des entreprises qui n'ont plus à trop se soucier de la proximité des fournisseurs ou des débouchés pour leur localisation est également présente dans une publication issue d'un colloque sur le développement en milieu rural ou péri-urbain tenu en Nouvelle-Ecosse (Coffey et Runte, 1986). C'est là un des éléments mis en évidence par Denis Maillat dans une communication sur les initiatives locales qui contribuent au redéploiement des activités de l'Arc jurassien. Pour sa part, Mario Polèse montre que la concurrence entre les régions repose de plus en plus sur les avantages des unes et des autres en matière d' information et de savoir faire. Leur dotation en ressources naturelles, aussi importante soit-elle, se fait moins déterminante. Conformément à certains points de vue déjà signalés, l'auteur, à partir de l'exemple d'une zone de pêche, soutient que le développement de l'économie locale exige bien plus que l'abondance de poisson. Les agents économiques de la région se doivent de posséder les connaissances, les moyens techniques et le savoir faire pour mettre en valeur les ressources et assurer leur mise en marché.

De telles idées se retrouvent également dans un numéro spécial de Plan Canada intitulé Rural Planning/Aménagement rural. Plus particulièrement, David Douglas (1989) présente un article très fouillé : Community Economic Development :ACritical view. Ce professeur de l'Université de Guelph suggère quelques éléments de définition : «Le développement économique communautaire émane de la conjugaison d'intérêts socio-économiques locaux. Il se distingue ainsi de l'ensemble des décisions prises sur une base individuelle sous l'effet des incitations du marché»". En plus, l'auteur identifie un certain nombre de caractéristiques particulières au développement local et fait part de modeles de gestion déjà mis en pratique par certaines communautés rurales. Sur la base de sa formation d'urbaniste, il propose des avenues possibles et des défis à la fois pour les communautés rurales et pour les gens de sa profession. Malheureusement, ces publications, en se rapportant au milieu rural, ne contribuent pas à lever l'ambiguïté que semble traîner comme un boulet le concept de développement local.

\section{Conclusion : pour en finir avec l'ambiguïté}

L'abondance de publications sur le sujet devrait permettre de clarifier le concept de développement local de façon à ce que les uns et les autres, en s'y référant, évoquent les mêmes réalités. On n'en est hélas pas encore là. Et les efforts de Xavier Greffe (1988), pour qui le développement local ne s'est jamais débarrassé du "paradigme de l'économie de clocher», ne suffisent pas. Pourtant les choses évoluent. Depuis quelques années, en France, l'Association nationale pour le développement local et des pays consacre une attention toute particulière au développement local urbain. Cinq mille kilomètres à l'ouest, à Montréal plus précisément,

* Traduction de l'auteur. 
le développement local est devenu une réalité concrète même si, en termes de créations d'emplois et d'entreprises, les résultats des différents efforts demeurent modestes, comme le fait voir Pierre Hamel (1990). Il apparaît, en conséquence, impertinent de partager l'histoire récente du développement local en deux catégories comme le fait $X$. Greffe. La première se voudrait une alternative à l'économie dominante, caractérisée par une idéologie anti-marché. La seconde, en réaction à la crise de la fin des années 1970, miserait essentiellement sur la création d'entreprises; d'où la ferveur actuelle envers l'entreprenariat. Or, la première (genre «fromage de chèvre» ou communautés sylvo-agraires à la recherche de l'autarcie) ne touche plus qu'une infime minorité d'anciens «soixante-huitards» sans influence. Dès la fin des années 1970, la majorité de ceux pour qui l'étiquette «alternatif» convient toujours, ont cessé de tourner le dos au mécanisme du marché; ils ne dédaignent plus les principes de gestion qui contribuent à la rentabilité d'une entreprise et agissent dans un cadre partenarial. Par ailleurs, $X$. Greffe a raison de présenter le développement local comme une méthode d'approche à défaut d'être un modèle bien articulé. Tel qu'indiqué au début, l'allusion à une confusion possible entre le développement local et la création de petites entreprises s'avère également pertinente.

Enfin, avec l'ouvrage plus récent de Bernard Pecqueur (Le développement local, 1989), il y avait lieu de croire à l'obtention d'un meilleur éclaircissement sur le concept. Surtout quand cetauteur signale que la documentation sur le développement local, bien souvent, ne dépasse pas l'approche monographique, rendant ainsi difficile la mise en évidence d'une cohérence d'ensemble. Il traite surtout - et ce, de façon, par ailleurs, fort intéressante - des systèmes locaux. Or cet ouvrage, malgré son titre, ne fait pas avancer le débat sur le concept du développement local.

Néanmoins, à la faveur de tout ce qui s'écrit, les acteurs comme les observateurs du «local» devraient mieux comprendre la portée exacte d'une stratégie qui, on doit le reconnaître, n'est pas dépouvue de «Monsieur Jourdain».

\section{BIBLIOGRAPHIE}

Arocena, J., (1986), Le développement par l' initiative locale, Paris. L'Harmattan.

Blakeley, E.J.,(1989), Planning Economic Development:Theory and Practice, Londres, SAGE Pu.

Cahiers (Les) du Laboratoire d'Etudes et de Recherches Appliquées en Sciences sociales (LERASS) (1990), no 20, LERASS-IUT, Toulouse.

Coffey,W., et Runte, R. (éd.), (1986), Le développement local : l'avenir des communautés culturelles isolées et des petites régions économiques, Pointe-de-l'Église, Presses de l'Universite Sainte-Anne.

Collectif, (1987), Initiatives et Solidarités :l' affaire de tous, Comptes rendus de la «Deuxième rencontre d'Aurillac», Paris, Syros.

Collectif, (1988), Le projet rural, Paris, L'Harmattan.

Commission des Communautés Européennes, (1989) Réflexions sur les Aspects sociaux du marché intérieur : une autre dimension de l'Europe, Bruxelles. 
Dionne, H., (1989), «Le développement local villageois comme projet de société», dans Le local en mouvement, GRIR, Université du Québec à Chicoutimi.

Douglas, D., (1989), «Community economic development», Plan Canada, vol 29, no 2.

Gidel, (1989), «L'apport au développement économique local», Rapport d'étape remis au ministre délégué, chargé de l'Aménagement du Territoire et des Reconversions, Paris.

Greffe, X., (1988)Décentraliser pour l' emploi : les initiatives locales de développement, Paris, Economica.

Hamel, P., (1990), «Le développement local en milieu urbain», Coopératives et Développement, vol. 21, no 2.

MacLeod, G., (1989), dans L'autre économie; une économie alternative ? Sous la direction de B. Lévesque, A. Joyal et $\mathrm{O}$. Chouinard, Québec, Presses de l'Université du Québec.

O.C.D.E., Innovation et Emploi , OCDE, Programme ILE, 2 rue André Pascal, 75775 Paris Cedex 16. FF : 170/Ecu : 25.

Pecqueur, B., (1989), Le développement local, Paris, Syros Alternative. 